\title{
4 Collaborative relaying in downlink cellular systems
}

C. Raman, G. Foschini, R. Valenzuela, R. Yates, and N. Mandayam

\subsection{Introduction}

The deployment of relays in cellular system has recently been standardized in the WiMAX, IEEE 802.16j standard and is a topic of discussion in the advanced specifications of 3GPP-Long Term Evolution (LTE) [2]. Although commercial relay deployments in cellular systems are not prominent at present, future wireless cellular systems will involve operation with dedicated relays for improving coverage, increasing cell-edge throughput, delivering high data rates and assisting group mobility. The proposed architecture is such that relays would be placed at certain locations (planned or unplanned) in the cell to help in forwarding the message from the base station to the user in the downlink, and from the user to the base station in the uplink. Relays will be more sophisticated than simple repeaters and could perform some digital base band processing to help the destination terminal get better reception. These relays will rely on air interfaces, and hence avoid the considerable back haul costs involving data aggregation and infrastructure costs associated with backbone connectivity. However, there are a lot of open issues that require research to answer. We present some of these issues in the sequel:

\section{Throughput gains due to relay deployments}

In cellular networks that are coverage limited, deploying relays can help in multihop transmission and provide power gains due to reduction of distance attenuation [3]. These power gains, in turn, translate to throughput improvements for the edge users. However, in interference limited settings, as is common in cellular systems, uncoordinated transmission by relays lead to increase of the overall interference levels in the cell and could be counter-productive by reducing the signal-to-interference plus noise (SINR) levels of users in the system. Coordination of transmissions in the system would require centralized control and incur high costs and overhead, especially in the uplink.

Thus, there is a need for a thorough evaluation of throughput improvements in a cellular system. In the cellular systems literature, there have been simulation studies to evaluate throughput gains in cellular systems, e.g., [7, 8, 9]. Even though the studies were conducted under different sets of (idealized) assumptions, throughput improvements in interference limited cellular systems are 
shown to be around $30 \%$ to $40 \%$ for the edge users. In this chapter, we evaluate the gains due to relay deployment by two different relaying strategies and the results indicate that throughput gains are of the same order. However, there may exist better practical schemes — which remain open — or specific scenarios where relays provide larger throughput improvements. A simple case where relays provide throughput improvements is the downlink scenario where the edge user is in a deep canyon and the relay is placed in the line of sight of both the base station and the shadowed user.

\section{Relay placement}

The benefits from relay deployment depend on where the relays are placed in the cell. Throughput improvements depend on the transmit power, relay antenna pattern and location of the relays in the system. Placing relays closer to an edge user helps the edge user. However, when relay transmissions are uncoordinated, the relays may cause near line-of-sight interference to an edge user of the neighboring cell. The optimal relay placement depends on the transmission and scheduling strategies, transmit power of the relays etc. A closely related issue to the relay placement problem is the choice of height of the deployed relays. In macro-cellular environments, propagation characteristics of the base-relay link and the relay-user link could be completely different, depending on whether the relays are mounted on tall poles or on roof tops. These factors may very well affect the system performance due to relay deployments. There are not many measurement based models to cover all the scenarios of relay placement; some empirical models were described in [1]. These issues apart, service providers often do not have much choice in placing the relays in a given geographical area.

\section{Lack of good models for relaying in cellular systems}

Multihopping in wireless networks has been studied in the context of ad hoc networks and peer-to-peer networks [5]. The main issue addressed in such networks is the routing problem. Interference constraints are abstracted as combinatorial constraints and many insightful results and good algorithms have been proposed to improve the throughput of such networks. Cellular networks, however, are unique in that the traffic is one-to-many in the downlink and many-to-one in the uplink. Direct application of the solutions obtained in the context of ad hoc networks are not optimal for cellular systems. Hence, performance evaluation of relays in cellular system requires fresh thinking into the problem.

On the other hand, the information theoretic relay channel [10] has been an active area of research for three decades now. But for some coding strategies proposed by Cover and El Gamal in [11] for special cases of the single relay channel, the capacity of the general relay channel is still unknown [12]. Though most of the earlier work assumes that the relay can transmit and listen over the same band, the half-duplex constraint (the relay cannot simultaneously 
transmit and receive at the same time in the same band) is taken into account in later work, for example $[13,14]$. The information theoretic studies reveal that when there are one or two relays, the best strategy is to make use of both the source and relay transmissions at the user location, rather than multihopping from the source to the destination through the relay(s). The intuition is that the user can make use of signals from both the source and the relay to get a better signal strength and hence a better rate. Multihopping on the other hand, ignores the signal from the source, however strong it is.

The information theoretic relaying protocols mentioned above often involve complicated multiuser coding and decoding techniques, that are too far from practical implementation. There have been some recent works trying to bridge the gap between the information theoretic and practical multihopping schemes, e.g., $[15,16]$. Most of the results in these works correspond to the case of a linear network of nodes, where there is a single commodity flow of message from the source node to sink node through a set of relay nodes. Any interference is only due to simultaneous transmissions from different relay nodes. This can be completely eliminated, by multiuser coding/decoding techniques. Such analysis does not carry over directly to the cellular systems since there are multiple simultaneous flows and multiuser techniques may incur significant overhead.

\section{Fairness}

Service level agreements of cellular service providers entail certain fairness requirements. For example, in cellular system, the edge users and the users near the cell require the same level of service. Many other fairness schemes including proportional fairness [18] and max-min fairness [19, Chapter 6] have been proposed for cellular systems serving voice and data. Present day cellular systems implement schedulers in the MAC layer to provide various degrees of fairness to users. In this work, we assume that the $90 \%$ of users are required to be served at a common rate. When relays are present in the system, designing distributed scheduling schemes to provide fairness is an active area of research.

In this chapter, we evaluate the performance of low-cost half-duplex relays in the downlink of a cellular system. The deployment scenario we consider is to mount a low-cost (preferably low-powered) device per sector over roof-tops of buildings. Such devices can relay the information from the base station to users in the cell.

\subsection{Related work and overview of our contribution}

Relay deployment in a cellular system has been proposed to solve the issue of lack of coverage over a large area [20]. The use of relays in cellular systems has also been proposed to bring capacity improvements [21]. Viswanathan et 
al. [7] studied the performance of a centralized throughput-optimal scheduler on a cellular network with relays. They present a centralized downlink scheduling scheme that guarantees the stability of user queues for the largest set of arrival rates into the system. Each user has a queue at the base station and at its serving relay and the objective of the scheduler is to stabilize both queues while maximizing the throughput. The throughput results obtained by simulations in [7] suggest that simultaneous transmissions (due to multihopping) exploiting spatial reuse could lead to cell-wide throughput gains in a cellular network.

In addition to a multihopping model, wherein the message travels to the destination in two hops, in this work we evaluate the performance of a collaborative power addition (CPA) scheme with a single relay available per user. We bring an additional dimension to the benefits of relays in a cellular system, by quantifying the power savings due to deployment of relays. Peak power savings in cellular networks are very important elements of amplifier costs in base stations. Significant peak power savings can reduce the cost of amplifiers and hence capital expenses for deploying cellular networks. Also, average power savings while operating cellular networks can save operational expenses such as electricity bills for the cellular operators.

In the first P-CPA scheme, we first consider a hypothetical model where $90 \%$ of the users are required to be served a file (henceforth, we use the term message and file interchangeably) of a fixed size within a certain deadline. Depending on the interference seen by each user, the mutual information (MI) or the instantaneous "rate" of the users vary over time. Users leave the system as they get the complete file within the deadline. We assume facility of large computation and use an offline computation to find the worst $10 \%$ of users that would not be able to get the complete file within the deadline and discard them at the beginning. We run the real system without the users in outage. When relays are present in the system, we evaluate the peak power savings at the base station to deliver a file of same size to the same number of users in the system as when the base stations and relays are transmitting at their peak power limits. We also find the improvements in common rate for the users in the presence of the relays when the peak power of the base stations are fixed for both the baseline and the system with relays.

We then include the power control capability to the base stations and relays. We evaluate the power savings and throughput improvement in the collaborative power addition scheme (PC-CPA). For a desired common rate requirement for $90 \%$ of users in the system, we find the common peak power constraint in the baseline case and in the system with relays to guarantee the common rate. When the relays get the complete message, they collaborate with the base station to transmit the message to the users. Each time a relay becomes eligible to transmit, the optimal set of powers are found to satisfy the desired rate requirement. The set of users that violate the peak power constraint are discarded at the beginning. The improvements in common rate are also evaluated through a similar procedure. 
The second relaying scheme we evaluate is simple multihopping, wherein the base stations and relays transmit in orthogonal time slots. The baseline system is similar to the baseline in the CPA scheme described above. When relays are present in the system, we simulate a time-slotted system. For a common rate requirement for $90 \%$ of users in the system, the base stations transmit at peak power in odd time slots. The relays and users are in the receive mode. In the even time slots, the base stations are turned off and relays transmit to the respective users. The relays employ power control to target the remainder of the user population to provide the residual rate to the users. We describe the system in detail in the later sections.

We do not consider any multiuser scheduling gains, MIMO gains and any other complex interference mitigation techniques. Thus the gains shown in the network are purely due to the power gains at the user location due to the relay transmissions.

\subsection{The set-up}

Our work aims to evaluate the power savings and improvement in common rate among users due to relay deployments in a cellular system. However, to model and simulate all dynamics of a cellular system can be too complicated. In order to overcome such difficulties, we make some reasonable simplifying assumptions and take an idealized look at the model and operation of a cellular system in our work. In order to make a fair comparison, the assumptions are kept consistent across systems with and without relays. We consider a cellular system with idealized hexagonal cells with a base station at the center of each cell. The topology is shown in Figure 4.1. The first two tiers of interferers are considered and the activities of the farther tier of cells are mirrored by the center ring of 19 cells. The site-to-site distance (distance between any two base stations) is taken to be 1 mile. The cells are divided into 120 degree sectors, each sector illuminated by a base station antenna pattern given by

$$
A(\theta)=-\min \left(12\left(\frac{\theta}{\theta_{3 d B}}\right)^{2}, A_{\max }\right)
$$

where $A(\theta)$ is the antenna gain in $\mathrm{dBi}$ in the direction $\theta,-180 \leq \theta \leq 180$, $\min ($. denotes the minimum function, $\theta_{3 d B}=70$ degrees is the $3 \mathrm{~dB}$ beamwidth and $A_{\max }=20 \mathrm{~dB}$ is the maximum attenuation. The antenna gain pattern is shown in Figure 4.2.

At the receiving terminal (relay or user), the transmitted power undergoes attenuation due to the distance traveled and shadowing effects around the receiver. The propagation attenuation between a transmitting terminal (base station or relay) and a receiving terminal (relay or user) consists of the path loss and the shadowing component. At any receiving terminal, the transmitted 


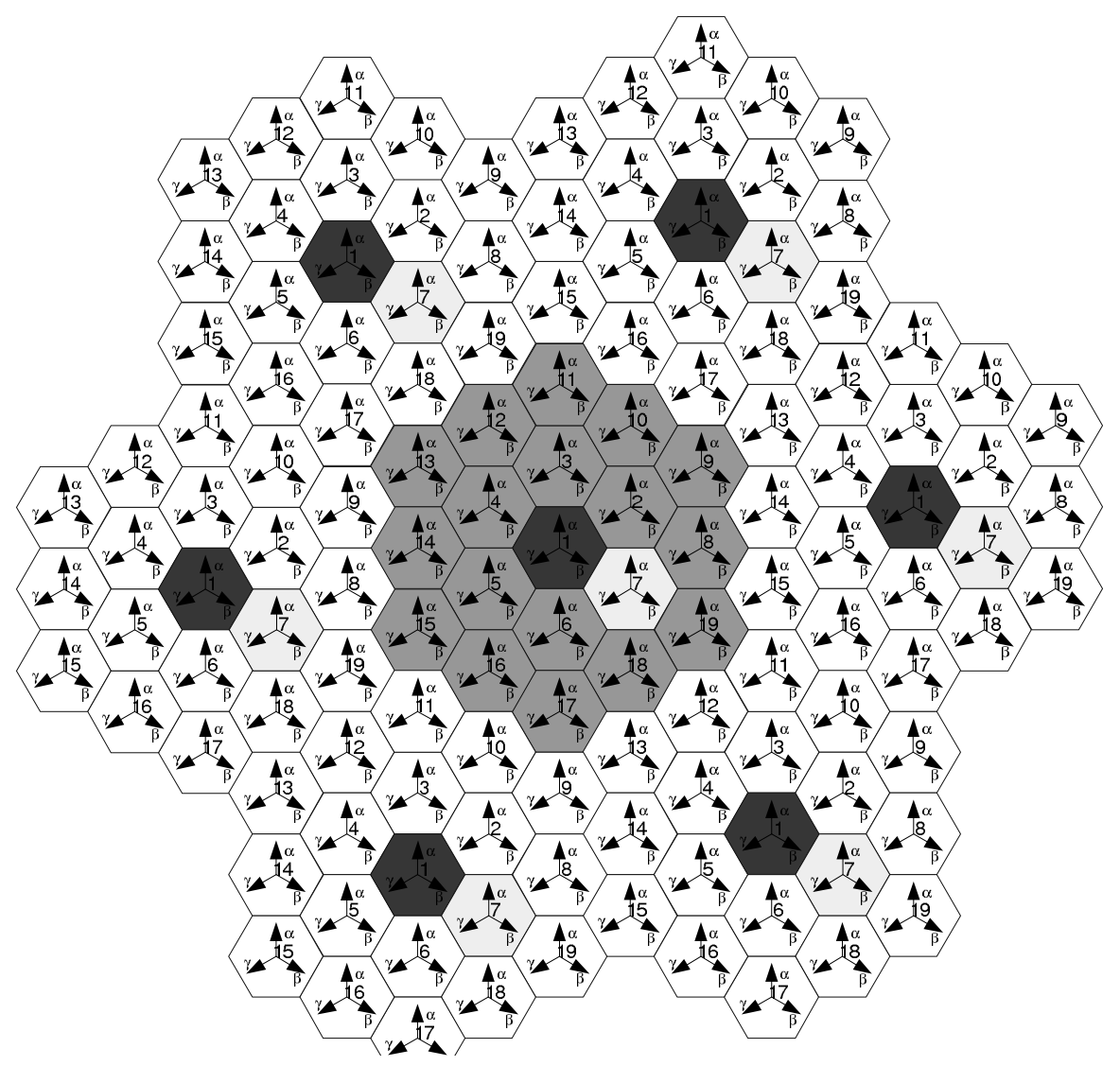

Figure 4.1: Wrap-around simulation model. The center ring of 19 cells are used for the simulation. The surrounding cell activity is mirrored in the center ring. The direction of the arrows represent the direction of the main lobe of the sectorized antenna.

power is attenuated in $\mathrm{dB}$ as $\mathrm{PL}(d)=-31.5-38 \log _{10} d$, where $d$ is in meters. The shadowing is modeled as lognormal with mean $0 \mathrm{~dB}$ and a standard deviation of $8 \mathrm{~dB}$. The shadowing is assumed to be spatially uncorrelated and fixed for a given set of user locations. The base station and the relay antenna gains are taken to be $15 \mathrm{~dB}$ (at zero degree horizontal angle) and user antenna gain as $-1 \mathrm{~dB}$. Other losses account for $10 \mathrm{~dB}$. Together with the above losses, we include the antenna pattern loss to calculate the received power. The receiver noise figure is set at $5 \mathrm{~dB}$, and the thermal noise power at the each receiving terminal (relay or user) is assumed to be $-102 \mathrm{dBm}$. The effect of multipath small scale fading is ignored in our simulations. 


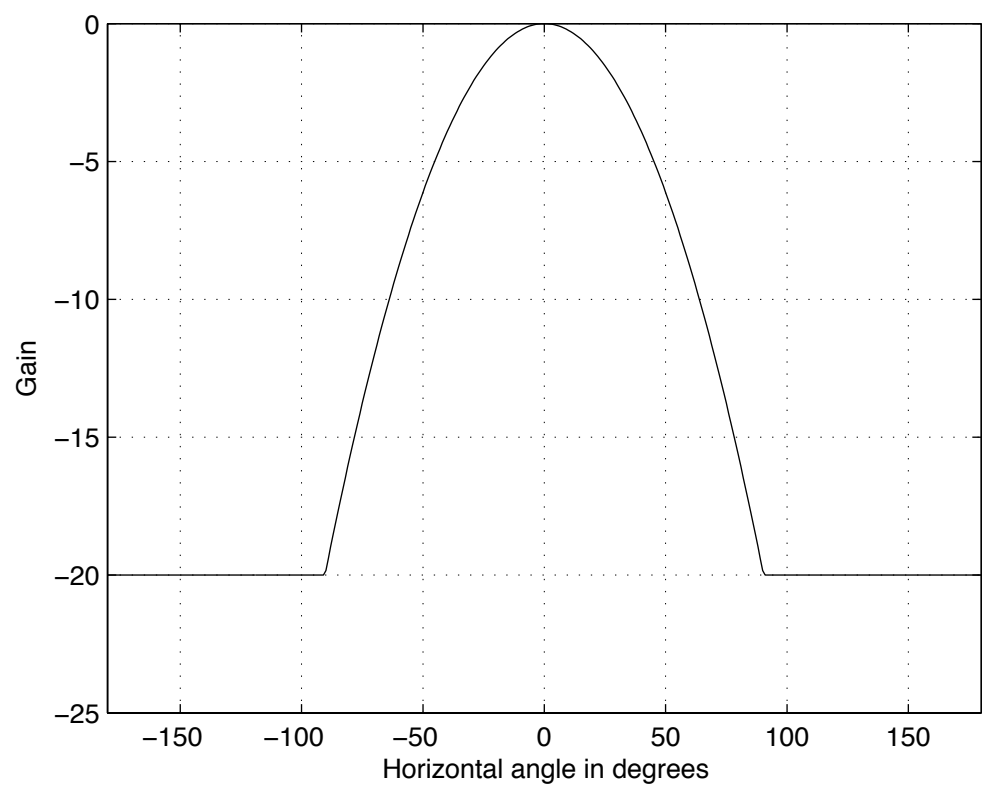

Figure 4.2: Antenna gain pattern (from [1]) as a function of the horizontal angle in degrees. The mathematical expression for the gain is given in equation (4.1).

All users share the same band of frequencies and hence simultaneous transmissions can interfere with each other. The total interference at each receiving terminal from all transmitters in the system is modeled as Gaussian noise and idealizes that other users use Gaussian codebooks. The achievable rate to a user $i$ at time $t$ is calculated as the Shannon rate

$$
R_{i}(t)=\log _{2}\left(1+\rho_{i}(t)\right)
$$

where $\rho_{i}(t)$ denotes the SINR for user $i$ at time $t$ The parameters used in the above mentioned simulation set-up are summarized in Table 4.1. We use this simulation set-up for evaluating all relaying methodologies proposed in this work.

\subsubsection{Placement of users and relays in the system}

We simulate a downlink OFDM-like system wherein users in orthogonal time or frequency slots do not interfere with each other. However, users in the same resource unit interfere with the other transmissions in the band. We simulate the worst case scenario where the system is fully loaded, i.e., users are present in all available resource units (or time-frequency slots) in all the sectors. The timefrequency slots are reused in each sector. We assume that the time-frequency slots are orthogonal, and focus only on a particular time-frequency slot within which 


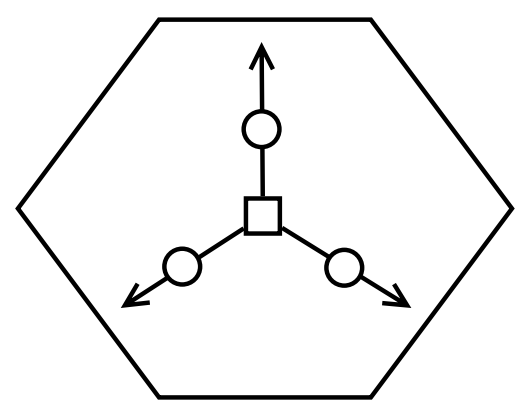

Figure 4.3: Position of relay location in a cell. The relays (represented by small circles) are placed at half the cell radius in the direction (given by the arrows) of the main lobe of the sector antenna. The base station at the center of the cell is represented by a square.

Table 4.1: Simulation parameters.

\begin{tabular}{ll}
\hline \hline Network Topology & 19 cells, 3 sectors per cell with wraparound \\
Site-to-site distance & $1 \mathrm{mile}$ \\
Bandwidth & $5 \mathrm{MHz}$ \\
Path loss model & COST-231 Hata model \\
Path loss exponent & $\alpha=3.8$ \\
Shadowing & Lognormal, with zero mean, $8 \mathrm{~dB}$ standard deviation \\
& for access and backhaul \\
Multipath fading & None \\
Antenna Pattern & Sectorized for base stations \\
& $\begin{array}{l}\text { Omnidirectional for relays } \\
\text { Antenna gains }\end{array}$ \\
& $-15 \mathrm{~dB}$ (for base station and relays) \\
Other losses & $10 \mathrm{~dB}$ \\
Thermal noise power at & $-102 \mathrm{dBm}$ \\
the receiver & $10 \%$ for baseline and relays \\
Outage & \\
\hline \hline
\end{tabular}

we simulate the complete cellular system such that there is one active user per sector at a given time. Hence, in a 19-cell network with 3 sectors per base antenna, at most 57 users are served at a given time-frequency slot. In our simulations, we use the following heuristic to create a random user population along with an association rule. Users are placed one-by-one in a uniformly random fashion across the network until all 57 base station sectors are occupied. For each random realization of a user location, the base station sector with the highest received signal strength is chosen to associate with the user. If the base station sector is already occupied by another user, the user is not allowed into the system 
and a new user location is generated. Along with a random realization of a user location, independent lognormal random variables also instantiated to account for the shadow fading gains between each base station and the user in the baseline system. If relays are present in the system, the fading gains are also generated for base station - relay links and relay - relay links. In this way, the random placement is carried out until all 57 sectors are occupied by exactly one user per sector. Each user is equipped with an omni-directional antenna.

A relay with an omni-directional antenna is placed in the direction of the main lobe of each base station sector antenna as shown in Figure 4.3. The relays always associate with the corresponding base station sector. The relay placement is an important parameter to be considered since the power gains and throughput improvements depend on the interference generated by the relays, which in turn, depends on the transmit power, geographic location of the relays and the propagation environment. In our simulations, we experiment with various relay placements and the simulation results are presented for the relay locations for which the gains are found to be maximum. The relay powers are also varied so that we get the maximum peak power savings.

\subsection{Collaborative relaying in cellular networks (CPA)}

In the Collaborative Power Addition (CPA) scheme devised in [22], the relay collaborates with the base station to help the message reach to the destination. In our simulation model, each base station sector has a single user to be served and a relay that may help the source to deliver the message to the user associated with the source. In what follows, we focus our attention on an isolated triplet of base station (source), relay and user in a single sector. Gaussian encoding is used across all other sectors, the interference from other sectors is considered as if it were additive Gaussian noise. Suppose the source wants to transmit one of $M$ messages to the destination, under a power constraint $P$. The source transmits a Gaussian codeword of length $N=(\log M) / R$, where $R$ is the rate of the code. By Shannon's channel coding theorem [23, Chapter 9], if $N$ is large enough, the message can be decoded reliably at the destination provided $R<\log (1+\rho)$, where $\rho$ is the received SINR. In our simulations, we are interested in achievable rates and assume that the instantaneous mutual information at the receiver is exactly $R=\log (1+\rho)$.

Assume that the source picks a rate $R$ code $\mathcal{C}_{1}$ and sends one of $M$ equally probable messages to the destination, using a codeword of length $N$. Let the received SINR $\rho_{S R}$ between the source and relay be greater than the received SINR $\rho_{R D}$ at the destination. Then, there exists some $\beta>1$ such that

$$
\log \left(1+\rho_{S R}\right)=\beta \log \left(1+\rho_{R D}\right)
$$

i.e., the capacity of the channel from source to relay is $\beta$ times greater than the channel from source to destination. We can now construct codebook $\mathcal{C}_{2}$ derived 


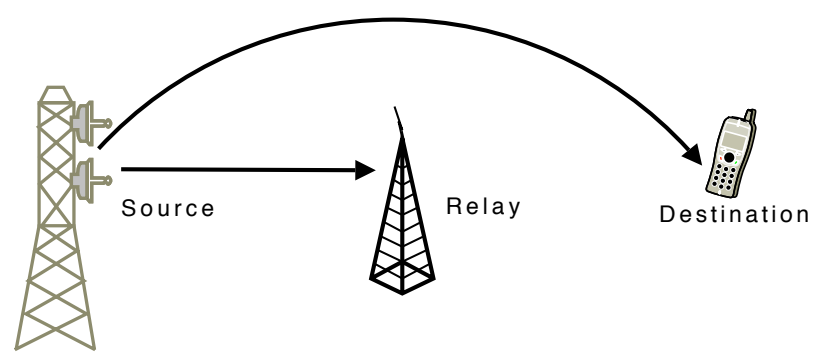

Figure 4.4: Collaborative relaying: Before relay decodes the message.

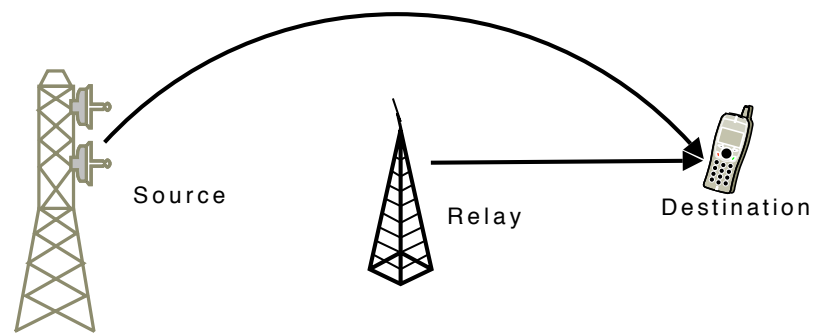

Figure 4.5: Collaborative relaying: After relay decodes the message.

from $\mathcal{C}_{1}$ by observing only the first $\lceil N / \beta\rceil$ symbols of every codeword. The relay can then reliably decode the received message since the rate of $\mathcal{C}_{2}$ is

$$
R^{\prime}=\frac{\log M}{\lceil N / \beta\rceil}<\log \left(1+\rho_{R D}\right) .
$$

In [24, Appendix F], the authors discussed the coding interpretation of a similar collaborative strategy. The authors also discuss the connection of such a coding setting with coding for arbitrary varying channel (AVC), which was first dealt with in [25] and then subsequently studied in [26]. We simulate a similar collaborative coding strategy wherein before the relay decodes the message as shown in Figure 4.4, the received power at the destination node is only due to the base station transmission. After the relay decodes the message, the relay joins the base station to help the base station in delivering the message to the destination as shown in Figure 4.5. At this point, if we assume that transmit symbol time slots at the relay and base station are synchronized and the code books are shared, the system can be viewed as a $2 \times 1$ MISO (Multiple-Input Single-Output) system without channel information at the transmitter. There is an effective power addition of the base station and relay transmissions at the destination [27, Chapter 3]. A similar scheme was proposed in the literature dynamic decode and forward (DDF) scheme [28].

We simulate this collaborative relaying strategy in two ways: 
- Base station and relay transmit at their respective peak powers. In this case, the transmit power is fixed and the users get variable rates depending on SINR at the user locations. When a target rate is obtained by a user, the user leaves the system and the corresponding base station sector is turned off, thus reducing the amount of interference in the system. We term this the peak collaborative power addition (P-CPA) scheme. This is described in Section 4.5.

- Base station and relay operate with power control so that the users obtain a target desired rate. In the baseline case, for a given desired rate requirement $r_{0}$ bps $/ \mathrm{Hz}$, a feasible set of powers are found to better satisfy the rate requirement, allowing for a certain users to be in outage. When the relays decode the message in the collaborative scheme, the optimal powers are recalculated to find another feasible set of powers to satisfy the rate requirement at the same outage level. We term this the power control collaborative power addition (PC-CPA) scheme. This is described in Section 4.6.

\subsection{CPA with peak power transmissions (P-CPA)}

\subsubsection{Principle of operation}

\section{P-CPA Baseline}

In the baseline of the P-CPA scheme, each base station sector transmits at its peak power to its own intended user. Since all users share the same band of frequencies, they observe interference from all the base station sectors in the system. If at time $t, p_{i}(t)$ is the peak power of the transmitting base station sector corresponding to the $i$ th user, and $h_{i j}$ is the channel gain, including path loss and shadowing, from the $j$ th base to the $i$ th user, and $\sigma^{2}$ is the variance of the noise power at the receiver, the instantaneous received SINR for user $i$ is given by

$$
\rho_{i}(t)=\frac{h_{i i} p_{i}(t)}{\sum_{j \neq i} h_{i j} p_{j}(t)+\sigma^{2}} .
$$

Since we assume Gaussian signaling, the MI (mutual information) or the instantaneous "rate" to each user is given as

$$
R_{i}(t)=\log _{2}\left(1+\rho_{i}(t)\right) \text { bits/symbol. }
$$

At time $t=0$, all base stations simultaneously transmit to their associated user. As time progresses, for any given time interval $[t, t+\Delta t]$, user $i$ accumulates MI $I_{i}(\Delta t)=R_{i}(t) \Delta t$. The MI for user $i$ at time $t$ is given by,

$$
I_{i}(t)=\int_{0}^{t} \log _{2}\left(1+\rho_{i}(\xi)\right) d \xi .
$$


If user $i$ accumulates MI corresponding to the required amount $L$ of data before the deadline $T$, i.e.,

$$
\tau_{i}=\min _{0 \leq t \leq T}\left\{t: I_{i}(t)=L\right\} .
$$

then the user leaves the system and his associated base station sector is turned off at time $\tau_{i}$, reducing the overall interference levels in the system. Hence,

$$
p_{i}(t)= \begin{cases}P, & t<\min \left(\tau_{i}, T\right) \\ 0, & t \geq \min \left(\tau_{i}, T\right)\end{cases}
$$

where, $P$ is the peak power of the base station transmission. Note that the $\rho_{i}(t)$ of user $i$ and the rate $R_{i}(t)$ are time varying quantities. At time $t=T$, the users that remain in the system are those users that did not get the complete file. It is these remaining users that are ascribed to be in outage.

\section{P-CPA system with relays}

The operation of the P-CPA system with relays is as follows. The requirement is the same as the baseline case: to deliver a file of size $L$ to as many users within the time $T$. At time $t=0$, the base stations transmit at peak power to users associated with them. The relay node placed in the sector also receives the data sent to the user by the base station. If the relay gets the complete file before the user gets it, the relay can potentially be useful to the user by helping it get the message faster. On the flip side, the relay transmission can create additional interference for the other users in the system. In our simulations, we follow a myopic ${ }^{1}$ policy on whether to turn on the relay or not: the relay transmits at peak power to help its user only if the instantaneous sum-rate of the whole system increases by turning the relay on. The sum-rate of the system is calculated as the sum total of the instantaneous rates of the existing users in the system and is a natural system-wide metric to use in order to decide whether the relays should transmit or not. At every epoch, a relay gets the message, among the set of all relays that are eligible to be turned on, the myopic sum-rate metric is applied and those relays that increase the sum-rate are turned on to help the users in the system.

If the relay increases the sum-rate of the system, the relay is turned on and helps the user with a transmission reinforcing the same message as the base station using the code described in Section 4.4. If $q_{i}(t)$ is the power transmitted from the relay $i$ at time $t$ and $g_{i j}$ is the channel gain from the user $i$ to the relay $j$, the effective SINR at $i^{t h}$ user location when the relay is active is given by

$$
\rho_{i}^{\text {relay }}(t)=\frac{h_{i i} p_{i}(t)+g_{i i} q_{i}(t)}{\sum_{j \neq i} h_{i j} p_{j}(t)+g_{i j} q_{j}(t)+\sigma^{2}} .
$$

1 The policy is myopic since, at the time when the relay gets the message, the global optimal decision whether the relay should transmit or not is unknown. 
The instantaneous rate and the mutual information for user $i$ at time $t$ are given by

$$
\begin{aligned}
R_{i}^{\text {relay }}(t) & =\log _{2}\left(1+\rho_{i}^{\text {relay }}(t)\right) \\
I_{i}^{\text {relay }}(t) & \left.=\int_{0}^{t} R_{i}^{\text {relay }}(\xi)\right) d \xi .
\end{aligned}
$$

If $H_{i j}$ denote the channel gain from the $j$ th base station to the $i$ th relay,

$$
J_{i}(t)=\int_{0}^{t} \log _{2}\left(1+\frac{H_{i i} p_{i}(\xi)}{\sum_{j \neq i} H_{i j} p_{j}(\xi)+\sigma^{2}}\right) d \xi
$$

represents the cumulative MI at the relay at time $t$.

Suppose the relay $i$ becomes eligible to transmit at time $t$, i.e., $J_{i}(t)>L$, then denote the sum-rate of the system at time $t$ as a function of $q_{i}(t)$ as

$$
S R\left(t, q_{i}(t)\right)=\sum_{i} \log _{2}\left(1+\frac{h_{i i} p_{i}(t)+g_{i i} q_{i}(t)}{\sum_{j \neq i} h_{i j} p_{j}(t)+g_{i j} q_{j}(t)+\sigma^{2}}\right) .
$$

Then, the relay power at time $t$ is given by

$$
q_{i}(t)= \begin{cases}Q, & \text { if } J_{i}(t)>L, S R(t, Q)>S R(t, 0) \text { and } t<T \\ 0, & \text { otherwise }\end{cases}
$$

where $Q$ is the peak power constraint of the relays. Each user sees a time-varying SINR and the time-varying rate given by $R_{i}(t)=\log _{2}\left(1+\rho_{i}^{\text {relay }}(t)\right)$. As with the baseline case, for any interval of time $[t, t+\Delta t]$, user $i$ accumulates MI amounting to $I_{i}(\Delta t)=R_{i}(t) \Delta t$ and the MI for user $i$ at time $t$ is

$$
I_{i}(t)=\int_{0}^{t} \log _{2}\left(1+\rho_{i}^{\text {relay }}(\xi)\right) d \xi
$$

Similar to the baseline case, if the user accumulates MI amounting to the full file size $L$ within the stipulated time $T$, the user leaves the system and the associated base station and relay are switched off. Thus the effective interference in the system is reduced. At time $t=T$, the users that remain in the system are those users that did not get the complete file.

\subsubsection{User discarding methodology}

The user discarding procedure can be divided in two phases:

(i) A learning phase where we learn the power threshold, that is used as a criterion to determine the users in outage. All the users that are not in outage require their corresponding base stations to have peak powers lower than the power threshold. The network will be operated with peak powers of all base stations are capped at the power threshold.

(ii) After the power threshold is found, we assume the availability of very fast computing facility and perform an off-line computation to find out the set 
of users that are in outage. Such users could be discarded upfront before the start of the simulations so that the others users benefit from the absence of interference from these users. Hence, this takes care of the causality of the discarding phase from the operation of the real network.

We conduct Monte Carlo simulation runs for the baseline case as well as the case with relays in our work. For each simulation run random instantiations of 57 user locations (as per Section 4.3.1) and associated statistically independent shadow fading values are generated. Once the random values are instantiated, these values are stored in our simulation software program. The same set of user locations and shadow fading values serve as inputs to the baseline and the system with relays.

We explain the learning phase now. Consider a single instance of the simulation runs in the baseline case. For the given instantiation, there are 57 users one in each sector. We fix a peak power threshold $P$ for the base stations and also fix the desired common rate for users as $r_{0} \mathrm{bits} / \mathrm{sec} / \mathrm{Hz}$. When the baseline system operation is over, the users that are in outage remain in the system at time $T$. Let the number of users in outage for the $k^{t h}$ instantiation when the power threshold is $P$ and desired common rate $r_{0}$ be $O_{k}\left(P, r_{0}\right)$. For the same power threshold $P$ and common rate $r_{0}$, we run a large number $K$ of instantiations. We then find the total number of users in outage as

$$
O\left(P, r_{0}\right)=\sum_{k=1}^{K} O_{k}\left(P, r_{0}\right)
$$

The percentage of users in outage for the threshold $P$ and desired rate $r_{0}$ is then

$$
\frac{O\left(P, r_{0}\right)}{57 K} \times 100 \% \text {. }
$$

If $O\left(P, r_{0}\right)>10 \%$, we increase the power threshold to $P^{\prime}>P$. On the other hand, if $O\left(P, r_{0}\right)<10 \%$, we decrease the power threshold to $P^{\prime \prime}<P$. Proceeding in this fashion, the base station peak power thresholds are adjusted such that exactly $90 \%$ of the users are guaranteed the desired rate of $r_{0} \mathrm{bits} / \mathrm{sec} / \mathrm{Hz}$ and the rest of the $10 \%$ users are in outage.

We could improve the performance of the system by discarding the users in outage upfront, since the interference due to the presence of these users will be eliminated at time $t=0$. In our simulations, for a large user population over $K$ instantiations, we identify $10 \%$ users in outage ${ }^{2}$ by first running $K$ instantiations of the system with all the users present in the system. We store the coordinates of all the users that were in outage at the end of each of the $K$ instantiations. We then eliminate the outage users from the system (by preserving the coordinates of the user locations of only those users not in outage for all the $K$ instantiations)

2 We remark that the eliminated set of $10 \%$ users in outage is not claimed to be the optimum set as would be obtained by evaluating all possible subsets amounting to $10 \%$ of the users. The latter is computationally prohibitive. 
at time $t=0$ in the real network simulations. Thus, the existing users in the system would experience lesser interference due to the absence of those users in outage when the real network is simulated.

\subsubsection{Network operation and simulation aspects}

Our objective is to obtain power savings and throughput improvement benefits due to deployment of relays in cellular system. To compare systems with and without relays in the CPA based relaying scheme, we simplify the operation of a cellular downlink system such that $90 \%$ of the users in the system are guaranteed to be delivered a file of fixed size $L$, within a fixed period of time $T$. The file could be different for all users but the file sizes are fixed. Such an operation brings in the notion of a common rate for the users in the system. In order that the system benefits from the users that get the message within the fixed time $T$, the satisfied users leave the system, thus no longer causing interference to the remaining users. The remaining $10 \%$ of the users that are not guaranteed of the file of size $L$ are ascribed to be in outage.

In our simulations, for the sake of simplicity, all base stations are assumed to have the same peak power threshold values. We run $K=200$ (amounting to 11400 user instantiations) different user instantiations in the system. The common rate requirement is set as $1 \mathrm{bit} / \mathrm{sec} / \mathrm{Hz}$. We divide the total time $T$ into 1000 mini-slots and at the end of each mini-slot, we keep track of the cumulative MI $I_{i}(t)$ of each user $i$. If at the end of a mini-slot, a particular user's cumulative MI exceeds the file size $L$, the base station corresponding to that user is turned off.

We run the baseline for different peak power values of the base station ( $5 \mathrm{~W}$ to $30 \mathrm{~W}$ in increments of $5 \mathrm{~W}$ ). For each peak power value, the relay powers are varied as a factor of the base station power. Figure 4.6 shows the variation of outage probability for various base station powers and various relay powers. For the case when there are no relays in the system (ratio of relay power to base station power is zero), increasing the peak powers of the base station decreases the outage. The percentage of outage saturates below a certain threshold as the interference limit sets in. As we increase the relay powers by increasing the ratio of relay power to base station power, the outage reduces but quickly saturates to a certain threshold outage value, because of the interference limit. From the Figure 4.6 it is clear that interference limit is quickly reached and limits the performance of system with relays. This is because we do not control the interference and peak power transmissions from the base stations and relays lead to a highly interference limited scenario. 


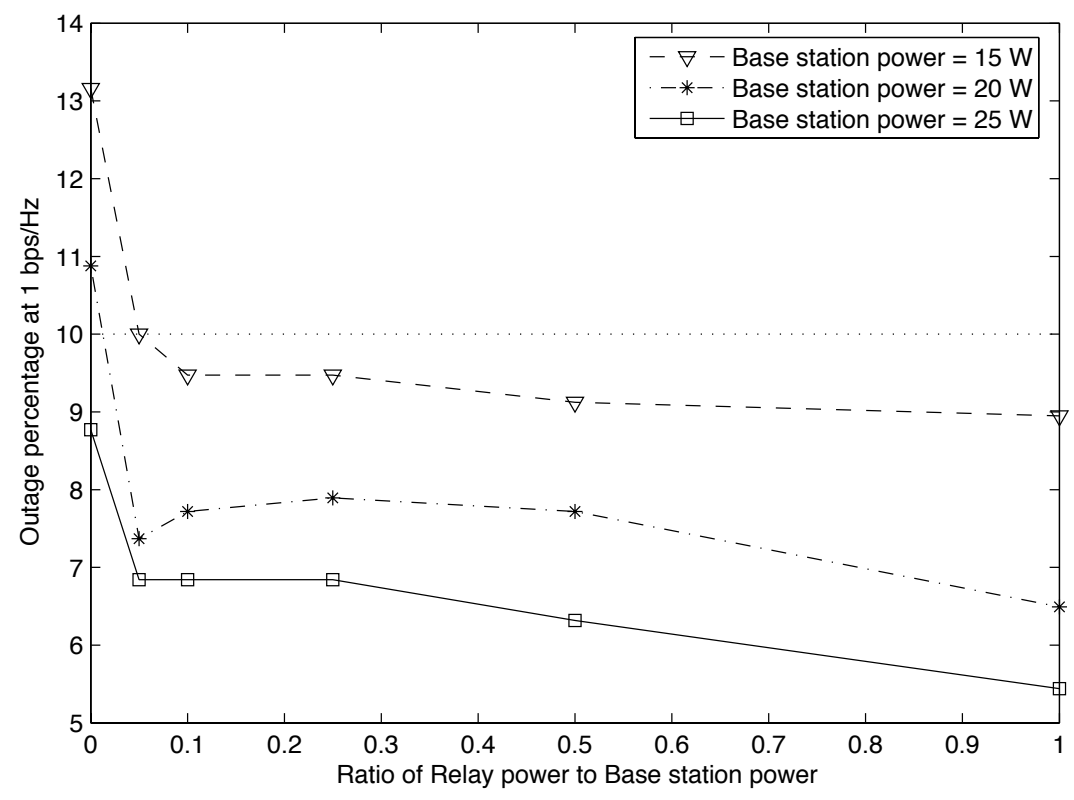

Figure 4.6: Variation of outage with relay powers and base station powers. As we increase the base station powers with no relays in the system $($ ratio $=0)$, the outage decreases and saturates at around $5 \%$, due to the inteference limit. The interference limit sets in very quickly even for smaller values of relay powers.

\subsubsection{Simulation results}

\section{Power savings}

The peak power required to guarantee $90 \%$ of the users (after the $10 \%$ users in outage have been removed) a rate of $1 \mathrm{bit} / \mathrm{sec} / \mathrm{Hz}$ is $21 \mathrm{~W}$ in the baseline case and it requires $15 \mathrm{~W}$ for a system with relays. The relays transmit $1 \mathrm{~W}$ of peak power. Hence the peak power savings at the base station locations in this case is $1.46 \mathrm{~dB}$ as shown in Table 4.2 .

\section{Rate gains}

In order to evaluate the throughput improvement, we find how much the common rate of $90 \%$ of users can be improved with the peak power of the base stations being fixed. For the baseline, we fix the power of the base stations to $21 \mathrm{~W}$, so that $90 \%$ of the users are guaranteed to get $1 \mathrm{bit} / \mathrm{sec} / \mathrm{Hz}$ (as obtained in the previous section). The $10 \%$ of the users in outage are eliminated as explained in Section 4.5.2. For the P-CPA system with relays, the peak power threshold of the base stations are fixed to $21 \mathrm{~W}$ (the same value as in the base line case). For the same peak power for the base stations and with relays present in the system, we 
Table 4.2: P-CPA relaying (base station and relays transmit at peak power).

\begin{tabular}{ccc}
\hline \hline $\begin{array}{c}\text { Peak power required to } \\
\text { guarantee } 1 \mathrm{bps} / \mathrm{Hz} \\
\text { at } 10 \% \text { outage } \\
\text { Baseline (No relays) }\end{array}$ & $\begin{array}{c}\text { Peak power required to } \\
\text { guarantee } 1 \mathrm{bps} / \mathrm{Hz} \\
\text { at } 10 \% \text { outage } \\
\text { With relays }\end{array}$ & Savings in dB \\
\hline $21 \mathrm{~W}$ & $15 \mathrm{~W}$ & 1.46 \\
\hline $\begin{array}{c}\text { Common rate for } 90 \% \text { users } \\
\text { Baseline (No relays) }\end{array}$ & $\begin{array}{c}\text { Common rate for } 90 \% \text { users } \\
\text { With relays }\end{array}$ & $\begin{array}{c}\text { Percentage rate } \\
\text { increase }\end{array}$ \\
\hline $1 \mathrm{bps} / \mathrm{Hz}$ & $1.21 \mathrm{bps} / \mathrm{Hz}$ & $21 \%$ \\
\hline \hline
\end{tabular}

expect the common rate to be better than $1 \mathrm{bits} / \mathrm{sec} / \mathrm{Hz}$. To find the improvement in common rate, we fix a desired common rate $r^{\prime}>1 \mathrm{bit} / \mathrm{sec} / \mathrm{Hz}$ and run the sytem with relays. If this desired common rate is feasible ${ }^{3}$, we double the desired common rate and run the simulations again. Else, if the desired common rate is infeasible, we fix the new desired common rate at half the difference between the highest feasible common rate and the lowest infeasible common rate and rerun the simulations. In this manner, we converge to the achievable common rate in the presence of relays. In our simulations, we find that the common rate can be improved to $1.21 \mathrm{bits} / \mathrm{sec} / \mathrm{Hz}$ in the $\mathrm{CPA}$ based relaying scheme. Hence the common rate improvement is $21 \%$.

\subsection{Power control based collaborative relaying (PC-CPA)}

In case of P-CPA relaying in Section 4.5.3, we observed that the interference from the other relays and base station sectors was limiting the peak power savings in the system with relays. The reason for that is when the relays transmit to help the users, they transmit with peak powers and hence increase the interference levels in the system. If we could find the optimal set of powers to transmit for the base station and the relays, we could reduce the overall interference levels in the system. This may improve the gains in the system.

In the following, we describe a framework for power control in the downlink of a cellular system with relays. When the relays are not present, downlink power control in a cellular system is well studied and understood [29]. When relays are present in the system, power control, if performed jointly at the base stations and relay locations, can provide power savings and throughput improvement. We describe the PC-CPA relaying scheme in the following sections.

3 The common rate is feasible if all the users present in the system are able to get the desired rate. 


\subsubsection{Principle of operation}

\section{PC-CPA baseline}

For the PC-CPA baseline, the aim is to deliver a desired common rate for $90 \%$ of the user population by employing a simple power control scheme. Each base station sector powers down its transmitted power within the peak power limitations so that $90 \%$ of users are guaranteed a desired common rate of $r_{0}$ bits $/ \mathrm{sec} / \mathrm{Hz}$. Since all users share the same band of frequencies, they observe interference from all the base station sectors in the system. We use a common subscript for a base station or a user in a particular sector. If at time $t, p_{i}$ is the power of the transmitting base antenna corresponding to the $i$ th user (we drop the argument $t$ ) and $h_{i j}$ is the channel gain, including path loss and shadowing, from the $j$ th base to the $i$ th user and $\sigma^{2}$ is the noise power at the receiver, the instantaneous SINR of the $i$ th user in the system is given by

$$
\rho_{i}(t)=\frac{h_{i i} p_{i}}{\sum_{j \neq i} h_{i j} p_{j}+\sigma^{2}} .
$$

Since the transmission use Gaussian codebooks, the corresponding instantaneous rate for the user $i$ is given by

$$
R_{i}(t)=\log _{2}\left(1+\rho_{i}(t)\right) \mathrm{bits} / \mathrm{sec} / \mathrm{Hz} .
$$

The set of feasible powers such that the users not in outage are guaranteed with a rate $r_{0}$ is obtained by solving for the feasibility of instantaneous rates subject to peak power constraints, specified by

$$
\begin{aligned}
\log _{2}\left(1+\rho_{i}(t)\right) & \geq r_{0} \\
\text { i.e., } \rho_{i}(t) & \geq 2^{r_{0}}-1 \\
\text { subject to } p_{i} & \leq p_{i, \max }
\end{aligned}
$$

for all users $i$ not in outage. In practice, each base station increases its power autonomously in small increments, until it hits the peak power limit or when the user associated with it attains the desired rate $r_{0}$. Users that make the power constraint to go active before attaining the desired rate are discarded. We simulate the system without the users in outage such that all users get the desired rate. Since the transmit powers of the base stations are such that all users get a common rate, none of the users leave the system.

\section{PC-CPA system with relays}

In the PC-CPA system with relays, $10 \%$ of users are discarded in the manner similar to the baseline system. At time $t=0$, the relays do not have the complete message required to relay to the user. Hence, the system starts out as it does for the baseline case. The base stations increase their powers autonomously in small increments targetting the users rates to increase. Users that do not meet the peak power constraints in (4.23) are eliminated one after the other. The remaining users get the desired rate without violating the peak power constraint at the base 
stations. While the base station transmissions are targetted to the users, the relay in each sector also listens to the transmission by the base stations. Depending on the channel conditions and coupling of interference from the adjacent sectors, the relays get their message at different points in time. When the relay in the sector decodes the message from the base station, the relay collaboratively helps the base station such that the user gets a rate corresponding to the total SINR from the relay and the base station. As described in Section 4.4, the code books at the base stations and relays are designed such that the mutual information at the receiver corresponds to the sum of received powers at the user location [22]. In order to maintain the common desired rate for all users, the relay and base station jointly adjust their powers so that the user gets the desired rate. This ensures that the base station and relay transmit just enough power to the user to obtain the desired rate.

Let $p_{i}$ denote the power transmitted by the base station sector $i$ at time $t$ and $h_{i j}$ be the channel gain, including path loss and shadowing, from the $j$ th base to the $i$ th user. Let $q_{i}$ be the power transmitted from the relay $i$ at time $t$ and $g_{i j}$ be the channel gain to the user $i$ from the relay $j$. Then, when the relay and base station transmit simultaneously, the effective SINR at $i^{\text {th }}$ user location when the relay is active is given by

$$
\rho_{i}^{\text {relay }}(t)=\frac{h_{i i} p_{i}+g_{i i} q_{i}}{\sum_{j \neq i} h_{i j} p_{j}+g_{i j} q_{j}+\sigma^{2}} .
$$

As with the baseline case, the set of feasible powers (for both base station antennas and relays) such that the users not in outage are guaranteed with a rate $r_{0}$ is obtained by solving for the feasibility of instantaneous rates subject to peak power constraints

$$
\begin{aligned}
\log _{2}\left(1+\rho_{i}^{\text {relay }}(t)\right) & \geq r_{0} \\
\text { i.e., } \rho_{i}^{\text {relay }}(t) & \geq 2^{r_{0}}-1 \\
\text { subject to } p_{i} & \leq p_{i, \max } \\
\text { and } q_{i} & \leq q_{i, \max }
\end{aligned}
$$

for all users $i$ not in outage. If we consider the transmit powers of the base stations and relays as variables of optimization, we have a total of $2 N$ variables, for $N$ base station sectors in the system. Thus, power control in cellular systems in the presence of relays gives us additional $N$ degrees of freedom to optimize over. The transmit powers in the system can be optimized to reduce the maximum peak power transmission in the system, reduce total energy in the system etc. In what follows, we assume that a central controller has the knowledge of the all the channel gains between the base stations as well as relays and the users. We explain ways to achieve various aforementioned objectives using linear program (LP) formulations. 


\subsubsection{Optimization framework}

\section{Minimizing the total instantaneous transmit powers}

We are interested in evaluating the benefits of relays in minimizing the total instantaneous sum power in the system while delivering the common rate $r_{0}$ with $10 \%$ of the users being omitted from the system. The practical benefit of minimizing the total sum of transmit powers in a cellular system is to save the energy costs in the network. Saving energy costs translate to saving electricity bills at the cell sites for the cellular service provider.

The desired common rate for the users is fixed at $r_{0}$ bits/symbol. We define $\mathcal{A}(t)$ as the set of all active relays at time $t$, i.e., the set of relays that have obtained the message and are ready to help the base station. $\mathcal{A}^{c}(t)$ denotes the complementary set of all inactive relays. For simplicity, we drop the argument and write $p_{i}$ and $q_{i}$ for the base station powers $p_{i}(t)$ and $q_{i}(t)$ at time $t$, respectively.

At a given time $t$, we solves the following optimization problem:

$$
\begin{array}{cl}
\min _{\substack{p_{1}, \ldots, p_{N} \\
q_{1}, \ldots, q_{N}}} & \sum_{i} p_{i}+q_{i} \\
\text { subject to } & \log _{2}\left(1+\frac{h_{i i} p_{i}+g_{i i} q_{i}}{\sum_{j \neq i} h_{i j} p_{j}+g_{i j} q_{j}+\sigma^{2}}\right) \\
& \geq r_{0}, i=1, \ldots, N \\
& 0 \leq p_{i} \leq p_{i, \max }, i=1, \ldots, N \\
& 0 \leq q_{i} \leq q_{i, \max }, i \in \mathcal{A}(t) \\
& q_{i}=0, i \in \mathcal{A}^{c}(t) .
\end{array}
$$

The solution to the optimization problem (4.29), $p_{i}^{*}, q_{i}^{*}, i=1, \ldots, N$ defines the powers $p_{i}(t)=p_{i}^{*}$ and $q_{i}(t)=q_{i}^{*}$ that are used at time $t$. The optimization problem (4.29) is an LP, since we can write the constraint (4.29b) as

$$
\frac{1}{2^{r_{0}}-1}\left(h_{i i} p_{i}+g_{i i} q_{i}\right)-\sum_{j \neq i}\left(h_{i j} p_{j}+g_{i j} q_{j}\right) \geq \sigma^{2}
$$

for $i=1, \ldots, N$. Rewriting (4.29) in vector form, we have

$$
\begin{aligned}
s^{*}(t)=\min _{\mathbf{p}, \mathbf{q}} & \mathbf{1}^{T}(\mathbf{p}+\mathbf{q}) \\
\text { subject to } & \mathbf{A p}+\mathbf{B q} \leq-\sigma^{2} \mathbf{1} \\
& \mathbf{0} \leq \mathbf{p} \leq \mathbf{p}_{\max } \\
& \mathbf{0} \leq \mathbf{q} \leq \mathbf{q}_{\max }
\end{aligned}
$$

where

$$
\mathbf{A}=\left(\begin{array}{cccc}
-h_{11} /\left(2^{r_{0}}-1\right) & h_{12} & \cdots & h_{1 N} \\
h_{21} & -h_{22} /\left(2^{r_{0}}-1\right) & \cdots & h_{2 N} \\
\vdots & \vdots & \ddots & \vdots \\
h_{N 1} & h_{N 2} & \cdots & -h_{N N} /\left(2^{r_{0}}-1\right)
\end{array}\right)
$$




$$
\mathbf{B}=\left(\begin{array}{cccc}
-g_{11} /\left(2^{r_{0}}-1\right) & g_{12} & \cdots & g_{1 N} \\
g_{21} & -g_{22} /\left(2^{r_{0}}-1\right) & \cdots & g_{2 N} \\
\vdots & \vdots & \ddots & \vdots \\
g_{N 1} & g_{N 2} & \cdots & -g_{N N} /\left(2^{r_{0}}-1\right)
\end{array}\right)
$$

and

$$
\begin{aligned}
\mathbf{p}(t) & =\left[p_{1}(t) \ldots p_{N}(t)\right]^{T} \\
\mathbf{q}(t) & =\left[q_{1}(t) \ldots q_{N}(t)\right]^{T} \\
\mathbf{p}_{\max } & =\left[p_{1, \max } \ldots p_{N, \max }\right]^{T} \\
\mathbf{q}_{\max } & =\left[q_{1, \max } \ldots q_{N, \max }\right]^{T}
\end{aligned}
$$

where, $q_{i, \max }=0, i \in \mathcal{A}^{c}(t)$. Solution to the above LP provides the optimal power values that minimize the instantaneous total power in the system. We take a myopic approach of minimizing the total sum power of the system at time $t$ in order to reduce the total average power transmission in the system. Each time a relay becomes eligible for transmission, the LP is solved to find the best set of powers by minimizing the instantaneous powers in the system. Note that in some cases, when a relay is eligible to help the base station, turning off the base station may be the optimal thing to do. This choice comes out as a solution to the optimization program.

\section{Minimizing the peak transmit power}

Minimizing the peak transmit power leads to peak power savings in the system. A practical benefit of peak power savings is the significant savings in the cost of power amplifiers for the cellular base stations. If by deploying low-power relays in the system, we save in the cost of the power amplifiers of the base stations, cellular operators could save in capital expenses. To this end, we solve the following optimization problem of minimizing the maximum instantaneous transmit powers at the base stations:

$$
\begin{array}{cl}
\min _{\substack{p_{1}, \ldots, p_{N} \\
q_{1}, \ldots, q_{N}}} & \max _{i} p_{i} \\
\text { subject to } & \log _{2}\left(1+\frac{h_{i i} p_{i}+g_{i i} q_{i}}{\sum_{j \neq i} h_{i j} p_{j}+g_{i j} q_{j}+\sigma^{2}}\right) \\
& \geq r_{0}, i=1, \ldots, N \\
& 0 \leq p_{i} \leq p_{i, \max }, i=1, \ldots, N \\
& 0 \leq q_{i} \leq q_{i, \max }, i \in \mathcal{A}(t) \\
& q_{i}=0, i \in \mathcal{A}^{c}(t) .
\end{array}
$$


Rewriting the above LP in vector form yields,

$$
\begin{aligned}
p^{*}(t)=\min _{\mathbf{p}, \mathbf{q}} & \alpha \\
\text { subject to } & \mathbf{A p}+\mathbf{B q} \leq-\sigma^{2} \mathbf{1} \\
& \mathbf{0} \leq \mathbf{p} \leq \mathbf{p}_{\max } \\
& \mathbf{0} \leq \mathbf{q} \leq \mathbf{q}_{\max } \\
& \alpha \mathbf{1} \geq \mathbf{p}_{\max }
\end{aligned}
$$

where $\mathbf{A}$ and $\mathbf{B}$ are given by (4.32) and (4.33), respectively.

\section{Improving the common rate}

As a corollary to the above approaches, if we keep the peak power constant across both the baseline and the system with relays, we can increase the common targeted rate in the system with relays. The problem of maximizing the common rate can be posed as an optimization program with the transmit powers of the base station and relays as the variables. A central controller then solves the optimization program:

$$
\begin{array}{cl}
\underset{p_{1}, \ldots, p_{N}}{\max } & r_{0} \\
\text { subject to } & \log _{2}\left(1+\frac{h_{i i} p_{i}+g_{i i} q_{i}}{\sum_{j \neq i} h_{i j} p_{j}+g_{i j} q_{j}+\sigma^{2}}\right) \\
& \geq r_{0}, i=1, \ldots, N \\
& 0 \leq p_{i} \leq p_{i, \max }, i=1, \ldots, N \\
& 0 \leq q_{i} \leq q_{i, \max }, i \in \mathcal{A}(t) \\
& q_{i}=0, i \in \mathcal{A}^{c}(t) .
\end{array}
$$

The optimization program can be viewed as a sequence of linear feasibility problems because constraint set is non-convex. We solve this program by an iterative approach. We start with a low easily achievable target rate $r_{0}$ so that the constraint set (4.37b)-(4.37e) is feasible. We increase the target rate in small increments until the constraint set becomes infeasible. In each step, we get a set of feasible power assignments. The last set of feasible power assignments is the solution to the optimization program. The method converges, since the iterations generate a bounded sequence of increasing rates.

\subsubsection{User discarding methodology}

In our simulations, we eliminate $10 \%$ of users (over a large number of user realizations) in the following way. The procedure is identical for both the baseline and the system with relays. We simply assume the same peak power constraints for all base stations across the network. We fix the peak power threshold $p_{\max }$ for each base station. Consider a single instantiation, where there are 57 users in 
the system. We increase the transmit power in all base stations in small incremental steps to improve the rate of the users in the system. As we go along, we discard the user associated with the base station whose power constraint goes active first. The base station is also turned off. This reduces the interference coupled with other users. Within the remaining set of users, we can increase the transmit powers further. We then discard the next user causing the power constraint to go active and continue in this fashion until all remaining users in the system are guaranteed the desired rate of $r_{0}$ bits $/ \mathrm{sec} / \mathrm{Hz}$, without violating the peak power constraints. This procedure is repeated for a large number $K$ of users instantiations. Let the number of users in outage for the $k^{\text {th }}$ instantiation when the power threshold is $p_{\max }$ and desired common rate $r_{0}$ be $O_{k}\left(p_{\max }, r_{0}\right)$. We then find the total number of outage users for $K$ instantiations when the peak power threshold is $p_{\max }$ and the desired rate is $r_{0}$ is calculated as

$$
O\left(p_{\max }, r_{0}\right)=\sum_{k=1}^{K} O_{k}\left(p_{\max }, r_{0}\right)
$$

The percentage of users in outage for the threshold $p_{\max }$ and desired rate $r_{0}$ is then

$$
\frac{O\left(p_{\max }, r_{0}\right)}{57 K} \times 100 \%
$$

If $O\left(p_{\max }, r_{0}\right)>10 \%$, we increase the power threshold to $p_{\max }^{\prime}>p_{\max }$. On the other hand, if $O\left(p_{\max }, r_{0}\right)<10 \%$, we decrease the power threshold to $p_{\max }^{\prime \prime}<$ $p_{\text {max }}$. Proceeding in this fashion, the base station peak power threshold $p_{\max }$ is adjusted such that exactly $90 \%$ of the users are guaranteed the desired rate of $r_{0}$ bits $/ \mathrm{sec} / \mathrm{Hz}$ and the rest of the $10 \%$ users are in outage.

The coordinates of the discarded users are stored and the same set of users are discarded when relays are present in the system too. We remark here that the order in which the users are discarded results in different power levels from the base stations, due to variations in the interference coupling among the users. Hence depending on the peak powers limitations at the base stations, the order in which the users are dismissed should be chosen carefully.

\subsubsection{Network operation and simulation aspects}

\section{Baseline operation}

We operate the baseline system as well as the system with relays such that, over a large number of user loading iterations, $90 \%$ of users obtain a common average rate of $1 \mathrm{bit} / \mathrm{sec} / \mathrm{Hz}$. We follow the approach described in Section 4.6.3 to discard users in the system. For the PC-CPA baseline, we solve a series of linear feasibility problem to obtain the base station powers for guaranteeing the desired common rate. One after another, we discard users that would cause the peak power constraint to go active. Hence we find the feasible set of powers $p_{1}, \ldots, p_{N}$ for the baseline such that $90 \%$ of the users get exactly $1 \mathrm{bit} / \mathrm{sec} / \mathrm{Hz}$. 


\section{PC-CPA with relays: Average power savings}

The peak power constraint of the base stations are fixed at $p_{\max }$ such that the baseline can deliver $1 \mathrm{bit} / \mathrm{sec} / \mathrm{Hz}$ at $10 \%$ outage. Since the relays are assumed to be inexpensive, we assume small peak power constraints for the relays. In our work, the peak powers of the relays are fixed at $1 \mathrm{~W}$. Let us consider a single instantiation of 57 users in the system. We start off similar to the baseline after discarding the same set of users. The base stations target the users to deliver the common rate of $1 \mathrm{bit} / \mathrm{sec} / \mathrm{Hz}$. Only relays that have better SINR to the base stations than the user are eligible to help the user. The other relays are always inactive. At time $t=0$, all relays are inactive. The aim in this experiment is to maintain a constant rate of $1 \mathrm{bit} / \mathrm{sec} / \mathrm{Hz}$ throughout the course of the simulation. When relay $i$ is eligible to transmit at time $t$, we include relay $i$ into the set of active relays $\mathcal{A}(t)$ and solve the LP (4.29). We stop when all the eligible relays are included in the set of active relays. The total power in the system when all eligible relays are active is noted down for this instantiation. We repeat this experiment for all the $K$ instantiations.

\section{PC-CPA with relays: Peak power savings}

The peak power constraints of the base stations are fixed at a value smaller than the baseline, say $p_{\max }^{\prime}$. We assume inexpensive relays being deployed in the system. Thus, the peak power constraints of the relays are fixed at $1 \mathrm{~W}$. Since the peak power value of the base stations is reduced from the baseline and the common rate is fixed at $1 \mathrm{bit} / \mathrm{sec} / \mathrm{Hz}$, the outage will be more than $10 \%$. Let us consider a single instantiation of 57 users in the system. We start off similar to the baseline after discarding the same set of users. The base stations target the users to deliver the common rate of $1 \mathrm{bit} / \mathrm{sec} / \mathrm{Hz}$. Only relays that have better SINR to the base stations than the user are eligible to help the user. The other relays are always inactive. At time $t=0$, all relays are inactive. Let relay $i$ be eligible to transmit at time $t$. We include relay $i$ into the set of active relays $\mathcal{A}(t)$ and solve the LP (4.35). We stop when all the eligible relays are included in the set of active relays. We repeat this experiment for all the $K$ instantiations and the outage is calculated. If the outage is less than $10 \%$, the peak power of base stations is reduced to $p_{\max }^{\prime \prime}<p_{\max }^{\prime}$, else the peak power values of the base stations is increased to $p_{\max }^{\prime \prime}>p_{\max }^{\prime}$ and the above procedure is repeated until the outage is close to $10 \%$.

\subsubsection{Simulation Results}

\section{Power savings}

We observe that the average power savings averaged over $K=200$ instantiations are $3 \mathrm{~dB}$. We observe that the peak power savings in the downlink when power control is employed is close to $2.6 \mathrm{~dB}$. 
Table 4.3: PC-CPA based relaying (base station and relays employ power control).

\begin{tabular}{ccc}
\hline \hline $\begin{array}{c}\text { Peak power required to } \\
\text { guarantee } 1 \mathrm{bps} / \mathrm{Hz} \\
\text { at } 10 \% \text { outage } \\
\text { Baseline (No relays) }\end{array}$ & $\begin{array}{c}\text { Peak power required to } \\
\text { guarantee } 1 \mathrm{bps} / \mathrm{Hz} \\
\text { at } 10 \% \text { outage } \\
\text { With relays }\end{array}$ & Savings in dB \\
\hline $10 \mathrm{~W}$ & $5.5 \mathrm{~W}$ & 2.6 \\
\hline $\begin{array}{c}\text { Common rate for } 90 \% \text { users } \\
\text { Baseline (No relays) }\end{array}$ & $\begin{array}{c}\text { Common rate for } 90 \% \text { users } \\
\text { With relays }\end{array}$ & $\begin{array}{c}\text { Percentage rate } \\
\text { increase }\end{array}$ \\
\hline $1 \mathrm{bps} / \mathrm{Hz}$ & $1.34 \mathrm{bps} / \mathrm{Hz}$ & $34 \%$ \\
\hline \hline
\end{tabular}

\section{Rate gains}

We have also performed experiments in which the rate improvements (problem (4.37)), and we have observed 34\% improvement in the throughput for $90 \%$ users in the system, with the baseline system being served at $1 \mathrm{bit} / \mathrm{sec} / \mathrm{Hz}$. The results are summarized in the Table 4.3.

\subsection{Orthogonal relaying}

In the previous sections where we studied relaying with the CPA scheme, we saw that in an interference-limited setting, the improvement in throughput was limited by the interference due to multiple transmissions in the cell. In some cases the transmission from the base stations are redundant. For instance, for a user located at the edge of the cell, the received power from the base station could be weak and the base station's signal could be of little use. In that case, it might be better to turn the base station off since it could benefit the system overall in terms of reducing the interference levels. Moreover, the practical implementation of such collaborative schemes can be complex with the existing technology. Hence, we investigate how much gains due to collaborative addition can be obtained if we just do simple multihopping, where the base station transmits to the relay in one slot and then the relay passes on the message to the destination in the next time slot. In this section, we exploit the half-duplex property of the relays in downlink cellular system to stagger the transmissions of the base station and relays over two time slots. A natural way to operate these relays is to have them receive in one time slot and transmit in another time slot. This gives us a natural orthogonality in the transmission scheme. Henceforth, we term this scheme as orthogonal relaying. 


\subsubsection{Network operation and simulation aspects}

The simulation set up is the same as that described in Section 4.3. Unlike CPA schemes, where the relay can start transmitting immediately after it decodes the message, relays can start transmitting only at specific times in the orthogonal relaying scheme. The system is assumed to be synchronous and time is divided into equal slots. The baseline and the system with relays are operated as follows.

\section{Baseline}

The baseline system operates similar to the P-CPA baseline as described in Section 4.5.1. All base stations transmit with peak powers and the users are required to get a fixed sized file with a specified deadline. Satisfied users leave the system as soon as they get the file. The associated base station sector is turned off. The users that do not get the file are in outage. The peak transmit powers of the base stations are fixed such that $10 \%$ of the users over a large population of users are in outage. However, in the orthogonal relaying case, we do not discard the users in outage and rerun the simulations.

\section{System with relays}

When relays are present in the system, time is divided into slots of equal durations, which is half of the baseline system. The operation of the system is periodic with odd and even time slots recurring at regular intervals. The base stations transmit in the odd time slots and the relays transmit in the even time slots. The peak power of the base stations are fixed as in the baseline and the peak power of the relays are fixed as $1 \mathrm{~W}$.

- In the odd time slots, the base stations transmit at peak power. Relays are in receive mode in this time slot. The users and relays in each sector accumulate mutual information, depending on their channel qualities. If some of the users get the desired rate from the base station transmissions itself, those users are satisfied users and leave the system as soon as they get the desired rate. The corresponding base stations and relays are turned off. Let us denote the $57 \times 1$ vector of rates obtained by users in the odd time slots by $\mathbf{r}_{o}$.

- In the even time slots, only the users that are yet to get the desired rate of $1 \mathrm{bps} / \mathrm{Hz}$ remain in the system. The base stations are turned off in this time slot. The relays that are required to help the users start transmitting simultaneously at the beginning of the even time slots. Simple power control is employed at the relay locations to reduce the interference caused to the other sectors. The power control is performed to achieve the desired residual common rate $\mathbf{r}_{e}=\mathbf{1}-\mathbf{r}_{o}$, where $\mathbf{1}$ is a $57 \times 1$ vector all 1's vector (representing $1 \mathrm{bps} / \mathrm{Hz}$ desired common rate). The users that require the relays to transmit more than their peak power constraint are discarded at the beginning of the even time slots. There may be cases where the user has a better channel to the base station than to the relay. Such users are not given the benefit of receiving the complete message from the base station. The base stations are 
switched off on the even time slots and are in outage if they do not get the message at the end of the even time slots.

\subsubsection{User discarding method}

\section{Baseline}

The users that remain in the system are in outage. However the users are not discarded in the baseline scheme.

\section{System with relays}

The user elimination procedure is same as that explained in Section 4.6.3. The users that violate the peak power constraint of the relays are discarded at the beginning of the even time slots. The discarded users do not get the desired common rate at the end of odd and even time slots, and hence are said to be in outage. The system is operated such that there is $10 \%$ outage in the system over a large number of user instantiations. The peak power threshold of the relay nodes are adjusted such that there the outage percentage is exactly $10 \%$.

\subsubsection{Simulation results}

The average power savings in the base station locations is $3 \mathrm{~dB}$, since the base stations transmit only for half the time. There is no peak power savings since the base stations transmit at peak power in the odd time slots. We obtain $35 \%$ rate gains due to orthogonal relaying when there is $10 \%$ outage in the system. It is interesting to note that simpler relaying methods, such as orthogonal relaying do nearly as well as the more complex forms of relaying, such as CPA schemes, in obtaining throughput gains and power savings. This observation is in agreement with the studies in simple linear settings [22, 30].

\subsection{Conclusion}

We have presented a simulation study of the downlink of cellular system with relays. We have evaluated the power savings and common rate increase for users when a common rate of $1 \mathrm{bps} / \mathrm{Hz}$ is required by $90 \%$ users in the system. We have first described the collaborative power addition (CPA) scheme of relay collaboration. In the CPA based scheme, whenever the relay gets the complete message from the base station, it collaborates with the base station such that the mutual information at the user location corresponds to the sum of the received power at the user location, thus boosting up the average rate. We observe that when the system is interference limited the peak power savings are hard to come by. Consequently, the power control based collaborative power addition (PC-CPA) scheme along with a framework for power control is proposed. The power control framework can be posed as a linear program formulation when 
Table 4.4: Summary of gains due to relaying.

\begin{tabular}{ll|cc}
\hline \hline Baseline & System with relays & \multicolumn{2}{c}{ Results } \\
\hline $\begin{array}{l}57 \text { sectors } \\
1 \text { user/sector }\end{array}$ & $\begin{array}{l}57 \text { sectors } \\
1 \text { user/sector and }\end{array}$ & $\begin{array}{c}\text { Power savings } \\
\text { (baseline at } \\
1 \mathrm{bps} / \mathrm{Hz})\end{array}$ & $\begin{array}{c}\text { Common rate gain } \\
\text { (baseline at } \\
1 \mathrm{bps} / \mathrm{Hz})\end{array}$ \\
\hline $\begin{array}{l}\text { Peak power } \\
\text { transmissions by } \\
\text { base stations }\end{array}$ & $\begin{array}{l}\text { Peak power transmissions } \\
\text { by base stations } \\
\text { and relays (CPA) }\end{array}$ & $1.46 \mathrm{~dB}$ (peak) & $25 \%$ \\
\hline $\begin{array}{l}\text { Base station } \\
\text { power control }\end{array}$ & $\begin{array}{l}\text { Base station and } \\
\text { relays power control } \\
\text { (PC-CPA) }\end{array}$ & $\begin{array}{c}2.6 \mathrm{~dB} \text { (peak) } \\
\text { (PB (average) }\end{array}$ & $34 \%$ \\
\hline $\begin{array}{l}\text { Peak power } \\
\text { transmissions by } \\
\text { base stations }\end{array}$ & $\begin{array}{l}\text { Relays power control } \\
\text { to users }\end{array}$ & $3 \mathrm{~dB}$ (average) & $35 \%$ \\
\hline \hline
\end{tabular}

the objective is to minimize peak power or to minimize average energy in the system. This formulation can be used to evaluate the average and peak power savings in the system. The peak power savings and the rate gains improve when power control is employed. We have then evaluated a simple multihopping scheme where the base stations and the relays transmit in orthogonal time slots. In the odd time slots, the base stations transmit at peak power and in the even time slots, the base stations are turned off and the relay employ simple power control to deliver the residual rate to the users. The summary of the results are given in the Table 4.4. 


\section{References}

[1] Multi-hop relay system evaluation methodology. http://ieee802.org/16.

[2] Y. Yang, H. Hu, J. Xu, and G. Mao, "Relay technologies for WiMAX and LTE-advanced mobile systems," IEEE Commun. Magazine, 47(10):100-105, October 2009.

[3] L. Le and E. Hossain, "Multihop cellular networks: Potential gains, research challenges, and a resource allocation framework," IEEE Commun. Magazine, 45(9): 66-73, September 2007.

[4] A. Goldsmith, Fundamentals of Wireless Communication. Cambridge University Press, Cambridge, UK, 2005.

[3] A. J. Viterbi, CDMA: Principles of Spread Spectrum Communication. Addison Wesley, 1995.

[4] Sergio Verdù, Multiuser Detection. Cambridge University Press, Cambridge, UK, 1998.

[5] Charles E. Perkins, Ad Hoc Networking. Addison-Wesley, 2001.

[6] R. H. Etkin, D. N. C. Tse, and H. Wang, "Gaussian interference channel capacity to within one bit," IEEE Trans. Inf. Theory, 54(12): 5534-5562, Dec 2008.

[7] H. Viswanathan and S. Mukherjee, "Performance of cellular networks with relays and centralized scheduling," IEEE Trans. Wireless Commun., 4(5): 2318-2328, Sept 2005.

[8] O. Oyman, J. N. Laneman, and S. Sandhu, "Multihop relaying for broadband wireless mesh networks: From theory to practice," IEEE Commun. Magazine, 45(11): 116-122, Nov 2007.

[9] O. Oyman, "Oppurtunistic scheduling and spectrum reuse in relay-based cellular OFDMA networks," in Proc. Globecom, 2007, Washington DC.

[10] E. C. van der Meulen, Transmission of Information in a T-terminal discrete memoryless channel. PhD thesis, Univ. of California, Berkeley, CA, 1968.

[11] T. M. Cover and A. A. El Gamal, "Capacity theorems for the relay channel," IEEE Trans. Inf. Theory, 25(5): 572-584, Sept. 1979.

[12] G. Kramer, M. Gastpar, and P. Gupta, "Cooperative strategies and capacity theorems for relay networks," IEEE Trans. Inf. Theory, 51:3037-3063, Sept 2005 .

[13] M. A. Khojastepour, A. Sabharwal, and B. Aazhang, "On the capacity of "cheap' relay networks," in Proc. 37th CISS, 2003. Baltimore, MD. 
[14] G. Kramer, "Models and theory for relay channels with receive constraints," in Proc. Allerton Conf. on Commun., Control, and Comp.,, 2004. UIUC, IL.

[15] D. Chen, M. Haenggi, and J. N. Laneman, "Distributed spectrum-efficient routing algorithms in wireless networks," IEEE Trans. Wireless Commun., 7(12):5297-5305, Dec 2008.

[16] M. Sikora, J. N. Laneman, M. Haenggi, Jr. D. J. Costello, and T. E. Fuja, "Bandwidth- and power-efficient routing in linear wireless networks," IEEE Trans. Inf. Theory, 52(6):2624-2633, June 2006.

[17] C. Raman, R. Yates, and N. Mandayam, "Cross layer scheduling of end-toend flows using a spectrum server," in Proc. CISS, 2006, Princeton, NJ.

[18] P. Bender, P. Black, M. Grob, R. Padovani, N. Sindhushayana, and A. Viterbi. "CDMA/HDR: A bandwidth-efficient high speed wireless data service for nomadic users," IEEE Commun. Magazine, 38(7):70-77, July 2000.

[19] D. Bertsekas and R. Gallager, Data Networks. Prentice-Hall, 1992.

[20] Harmonized Contribution on 802.16j (Mobile Multihop Relay) Usage Models. http://ieee802.org/16.

[21] R. Pabst, B. H. Walke, D. C. Schultz, P. Herhold, H. Yanikomeroglu, S. Mukherjee, H. Viswanathan, M. Lott, W. Zirwas, M. Dohler, H. Aghvami, D. D. Falconer, and G. P. Fettweis, "Relay-based deployment concepts for wireless broadband radio," IEEE Commun. Magazine, 42(9):80-89, Sept 2004.

[22] G. Foschini, A. Tulino, and R. Valenzuela, "Performance comparison for basic relay systems," Tech. Memo, Bell Labs NJ, 2008.

[23] T. M. Cover and J. A. Thomas, Elements of Information Theory. New York:Wiley, 2006.

[24] M. Katz and S. Shamai, "Transmitting to colocated users in wireless ad hoc and sensor networks," IEEE Trans. Inf. Theory, 51:3540-3563, Oct 2005.

[25] D. Blackwell, L. Breiman, and A. J. Thomasian, "Capacity of a class of channels," Annals of Mathematical Statistics, 30:1229-1241, 1968.

[26] I. Csiszar and P. Narayan, "Capacity of the gaussain arbitrarily varying channel," IEEE Trans. Inf. Theory, 37:18-26, Jan 1991.

[27] D. Tse and P. Viswanath, Fundamentals of Wireless Communication. Cambridge University Press, Cambridge, UK, 2005.

[28] K. Azarian, H. El Gamal, and P. Schniter, "On the achievable diversity-vsmultiplexing tradeoff in half-duplex cooperative channels," IEEE Trans. Inf. Theory, 51:4152-4172, Dec 2005.

[29] M. Chiang, P. Hande, T. Lan, and C. W. Tan, "Power control in wireless cellular networks," Foundations and Trends in Networking, 4(2):381-533, July 2008.

[30] N. Jacobsen, "Practical cooperative coding for half-duplex relay channels," In Proc. CISS 2009, March 2009. 